\title{
Avaliação da compreensão de rótulos de alimentos embalados por consumidores do município de Niterói, Rio de Janeiro, Brasil.
}

Paula Liberti

paulaliberti20@hotmail.com Centro Universitário Anhanguera de Niterói, Rio de Janeiro, Brasil.

\section{Gabrielle Ayres}

gabrielledsayres@gmail.com Rio de Janeiro, Brasil.

\section{Daise Coelho}

daisevcoelho@hotmail.com

entro Universitário Anhanguera de Niterói, Rio de Janeiro, Brasil.

Tatiana Lima

tatianalima costa@hotmail.com

Centro Universitário Anhanguera de Niterói Rio de Janeiro, Brasil.

Yasmin Fingola

yasminfingola@hotmail.com

Centro Universitário Anhanguera de Niterói Rio de Janeiro, Brasil.

Lucas Luquez

lucasrangelluquez@gmail.com

Centro Universitário Anhanguera de Niterói, Rio de Janeiro, Brasil.

\section{Nathalia Soares}

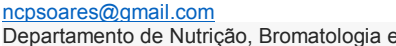
Tecnologia de Alimentos, Centro

Universitário Anhanguera de Niterói, Rio de Janeiro, Brasil.

\section{RESUMO}

O presente estudo teve por objetivo avaliar o entendimento de consumidores sobre as informações contidas em rótulos de produtos alimentícios comercializados em supermercados da cidade de Niterói, Rio de Janeiro, Brasil. Durante 90 dias do ano de 2016, 301 consumidores foram entrevistados, através de um formulário estruturado, sobre sua compreensão em relação à rotulagem nutricional. Os resultados mostraram que, dos entrevistados, $63,79 \%$ liam os rótulos de alimentos, sendo que, destes, somente $53,49 \%$ compreendiam as informações lidas. Os participantes, que não tinham costume de ler o rótulo de alimentos, declararam que a falta de hábito e a letra ilegível são dois importantes pontos para a não prática da leitura nutricional. Aos interessados no rótulo, o prazo de validade e a tabela nutricional são as informações mais lidas na embalagem do produto. Os consumidores demonstraram maior preocupação sobre informações como valor calórico, conteúdo de carboidratos e sódio; dados estes que tem relação com a busca da informação nutricional devido a problemas de saúde, como diabetes e hipertensão, conforme relatado pelos participantes. Além disso, para $63,46 \%$ dos consumidores, deste estudo, o marketing não tem influência sobre a decisão de compra de um produto embalado. Conclui-se que as informações contidas nos rótulos de alimentos são relevantes para o grande público, porém há a necessidade de projetos educativos no âmbito nutricional, de modo a facilitar a compreensão e melhor uso das informações descritas nas embalagens de alimentos.
\end{abstract}

PALAVRAS-CHAVE: Rotulagem nutricional; comportamento do consumidor; educação alimentar. 


\section{INTRODUÇÃO}

A rotulagem de alimentos é o meio de comunicação existente entre produtores e consumidores e, assim, a exatidão das informações nos rótulos nutricionais de alimentos deve ser segura e clara para auxiliar nas escolhas do cliente e nas orientações para a formação da dieta e melhorias na saúde (MACHADO et al., 2006). Para isso, os rótulos alimentares devem ser primeiramente fidedignos em relação ao conteúdo do produto e ter uma linguagem de fácil compreensão e escrita legível, pois a letra utilizada em muitas embalagens dificulta o correto entendimento dos consumidores, desmotivandoos na leitura das mesmas (YOSHIZAWA et al., 2003; LOBANCO et al., 2009). Nesse contexto, sabe-se que o marketing, que pode influenciar os consumidores nas suas aquisições de alimentos, e consequentemente nos seus hábitos de consumo, também tem se preocupado com as informações nutricionais e as alegações de saúde presentes nos rótulos dos alimentos e bebidas, embora nem sempre com um objetivo de melhorar a qualidade do consumo alimentar (COLBY et al., 2010).

A Agência Nacional de Vigilância Sanitária (BRASIL, 2002) na Resolução - RDC no. 259 , de 20 de setembro de 2002, regulamenta a rotulagem de alimentos, tornando obrigatório: a denominação de venda do alimento, a lista de ingredientes, o conteúdo líquido, a identificação da origem e do lote, o prazo de validade e a instrução sobre preparo e uso do alimento, quando necessário. Assim sendo, intervenções educativas precisam ser desenvolvidas para conscientizar a população sobre o valor da leitura dos rótulos de alimentos, e como tal atitude é valiosa para o conhecimento da composição do alimento e da segurança alimentar (SIQUEIRA et al., 2014). A instrução e informações corretas possibilitariam ao consumidor maior consciência na aquisição dos alimentos. Essa ação é imprescindível quando se verifica que a vulnerabilidade do consumidor está ligada à ausência de conhecimento técnico, pois, como ele não participa do ciclo de produção, acaba não tendo como avaliar o que comprar e decidir por ele mesmo qual produto é melhor (PONTES, 2009).

Diante do exposto, a demanda crescente da sociedade por informações confiáveis no que diz respeito aos produtos alimentares exige esforço do governo e setor produtivo para implantação de uma efetiva rotulagem nutricional de alimentos. Dessa forma, como parte do conjunto de indicações do Ministério da Saúde, a promoção de hábitos alimentares e estilos de vida saudáveis tem o compromisso de contribuir para a promoção e proteção da saúde da população. Assim, facilitar a escolha de alimentos saudáveis, a partir das informações contidas nos rótulos de alimentos, foi uma das estratégias desenhadas pela Política Nacional de Alimentação para a redução dos índices de sobrepeso, obesidade e doenças crônico degenerativas associadas aos hábitos alimentares da população (BRASIL, 2005b).

A educação alimentar e nutricional está vinculada à produção de informações que possam subsidiar a tomada de decisões por parte dos indivíduos, dando a eles o direito de optar por alimentos mais saudáveis (SANTOS, 2005). Por meio de uma alimentação equilibrada, o organismo humano adquire nutrientes que permitem um melhor desempenho e energia para realização das funções necessárias, além de contribuir para a prevenção de uma série de doenças crônicas nãotransmissíveis (SOUZA et al., 2011; MONDINI; MONTEIRO, 1994). Diante disto, a rotulagem nutricional torna-se uma ferramenta de educação alimentar. 
Deste modo, o objetivo do presente estudo foi determinar a proporção de consumidores com hábito de leitura e o conhecimento das informações nutricionais contidas nos rótulos de alimentos, bem como analisar os fatores que interferem nesse processo, por frequentadores de diferentes redes de supermercados do município de Niterói, Rio De Janeiro, Brasil.

\section{MATERIAIS E MÉTODOS}

Esta pesquisa tem caráter de estudo descritivo e de natureza transversal. Foram entrevistados 301 consumidores de supermercados do município de Niterói, Rio de Janeiro, Brasil, durante os meses de setembro a novembro do ano de 2016. Os supermercados foram escolhidos de forma aleatória dentro do município.

Os critérios de inclusão para participação na pesquisa foram consumidores maiores de 18 anos e de ambos os sexos. Os consumidores foram escolhidos aleatoriamente no ato de suas aquisições de alimentos e participaram voluntariamente, respeitando os critérios da pesquisa, tomando ciência dos objetivos do estudo e assinando o termo de consentimento livre e esclarecido. Para garantir a aleatoriedade do estudo, foram abordados os clientes que estavam na fila do caixa, aguardando o momento para pagar, sendo entrevistado o último cliente da fila, similarmente à metodologia descrita por SILVA (2003). O tempo médio de duração de cada entrevista foi de 15 minutos.

Para a coleta de dados foi utilizado um questionário semiestruturado adaptado de CASSEMIRO e cols. (2006), composto de treze perguntas fechadas, sendo respostas de múltipla escolha. Os participantes foram submetidos a perguntas referentes aos seguintes tópicos: variáveis socioeconômicas (gênero, idade, escolaridade e estado civil); hábito de leitura dos rótulos; compreensão das informações, e influência das informações na escolha dos produtos. Os dados foram coletados por entrevistadores treinados e as entrevistas foram realizadas nos dias úteis da semana, nos turnos manhã e tarde.

\section{RESULTADOS E DISCUSSÃO}

Na Tabela 1 é possível observar que o gênero feminino é predominante entre os entrevistados, com $79,07 \%$ de participação nesta pesquisa. Em relação ao grau de escolaridade, àqueles que possuem Ensino Médio completo totalizam pouco mais da metade dos entrevistados, atingindo um percentual de 50,50\%. Ainda na Tabela 1, nota-se que, dentre os participantes, $41,52 \%$ possuem como renda mensal o equivalente entre 2 e 3 salários mínimos.

Dos 301 entrevistados que participaram do estudo, 63,79\% ( $n=192)$ tinham hábito de ler as informações dos rótulos, e 36,21\% ( $n=109)$ não o faziam. Estes dados são semelhantes aos descritos por SIQUEIRA e cols. (2014) quando avaliaram o entendimento de consumidores com relação as informações veiculadas na rotulagem de produtos alimentícios no Estado do Espírito Santo. COSTA e cols. (2011) também apresentaram resultados similares, quando 
estudaram a utilização da informação nutricional dos rótulos por consumidores de Natal.

Tabela 1- Características da amostra (n=301). Niterói-RJ, 2016.

\begin{tabular}{ccc}
\hline Características & N & $\%$ \\
\hline Masculino & 63 & 20,93 \\
Feminino & 238 & 79,07 \\
\hline & Grau de escolaridade \\
\hline Não possui & 1 & 0,33 \\
Ensino fundamental completo & 38 & 12,62 \\
Ensino médio completo & 152 & 50,50 \\
Ensino superior completo & 110 & 36,55 \\
\hline & Renda salarial \\
\hline Até 1 salário mínimo & 83 & 27,58 \\
De 2 a 3 salários mínimos & 125 & 41,52 \\
Acima de 3 salários mínimos & 91 & 30,23 \\
Não responderam & 2 & 0,67 \\
\hline
\end{tabular}

Conforme demonstrado na Figura 1, dentre as causas da não prática de leitura dos rótulos alimentícios, pelos $36,21 \%$ de consumidores entrevistados que não cultivavam este hábito, estão destacadas as seguintes: falta de hábito $(40,87 \%)$, falta de interesse $(21,73 \%)$, falta de tempo $(20,00 \%)$ e letra ilegível $(17,40 \%)$. Como é possível observar, um fator que interferiu no interesse do consumidor em praticar o hábito da leitura do rótulo de um alimento foi a ilegibilidade da letra apresentada na embalagem. Diante disso, o direito assegurado no Código de Defesa do Consumidor, de clareza e adequação da informação sobre produtos e serviços, é ferido, podendo-se constatar uma contradição quanto à finalidade da rotulagem de alimentos, pois ao passo que esta representa um elo entre o consumidor e o produto, ela só é decifrada por aqueles que estão mais capacitados.

Em relação aos consumidores com a prática de leitura dos rótulos de produtos alimentícios, foi questionado a estes se os mesmos conseguem compreender o significado das informações descritas nos rótulos. $E$, apesar de $63,79 \%(n=192)$ dos consumidores responderem que apresentam o hábito da leitura dos rótulos, $38,87 \%$ destes entrevistados não compreendem as informações lidas (Figura 2). Vale ressaltar que o rótulo nutricional é um instrumento fundamental no momento da compra dos alimentos, uma vez que representa um elo de comunicação entre o consumidor e o produto, e, se o rótulo é bem compreendido, amplia o poder de escolha e decisão dos consumidores, garantindo, assim, condições para que possam exercer sua autonomia decisória na opção por escolhas alimentares mais saudáveis (SOUZA et al., 2014).

Outro ponto avaliado pelos consumidores, que apresentavam a prática da leitura de rótulos de produtos alimentícios, foi quanto a confiança das informações prestadas pela indústria de alimentos nestes rótulos. $\mathrm{E}$, conforme demonstrado na Figura 3, pouco menos da metade dos participantes, com hábito de leitura dos rótulos, possuem confiança sobre a veracidade das informações descritas na embalagem. 
Aproximadamente $38 \%$ dos entrevistados, nessa pesquisa, não confiam nas informações descritas nos rótulos, seja por acreditar que as informações são manipuladas, omitidas ou falsas, seja por achar que aquelas informações não sofrem fiscalização por parte dos órgãos competentes.

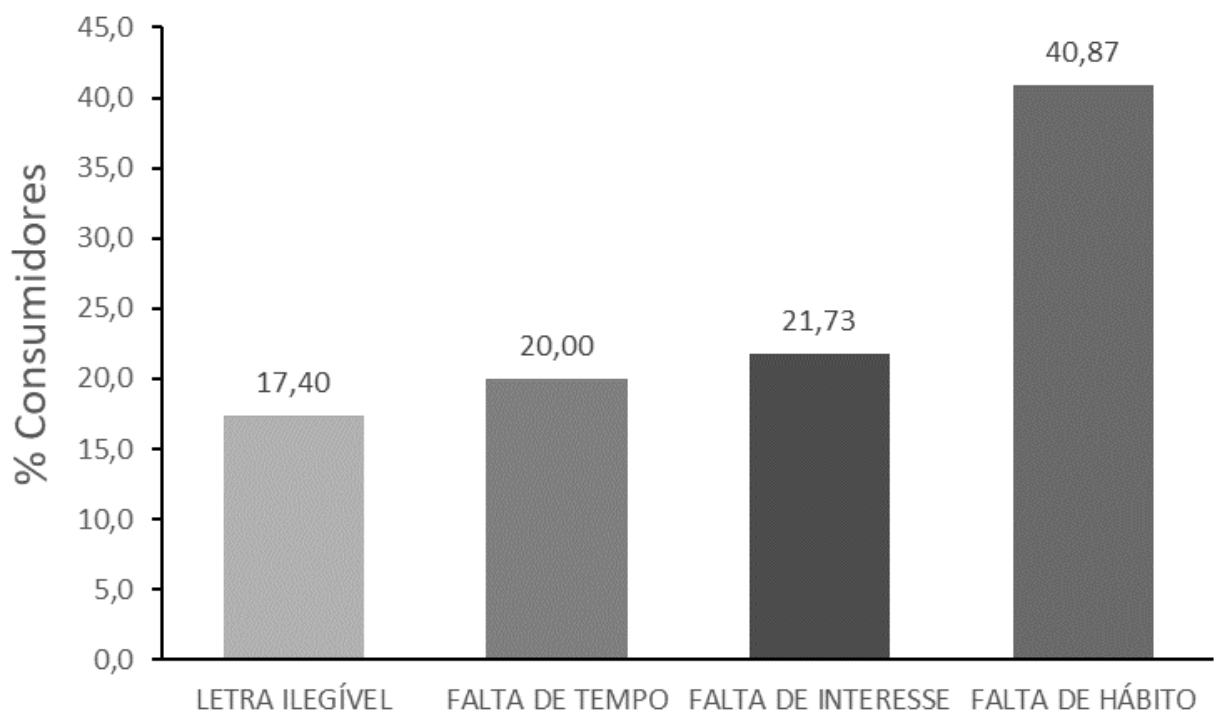

Figura 1- Causas da não prática de leitura dos rótulos alimentícios por consumidores de supermercados da cidade de Niterói-RJ, 2016.

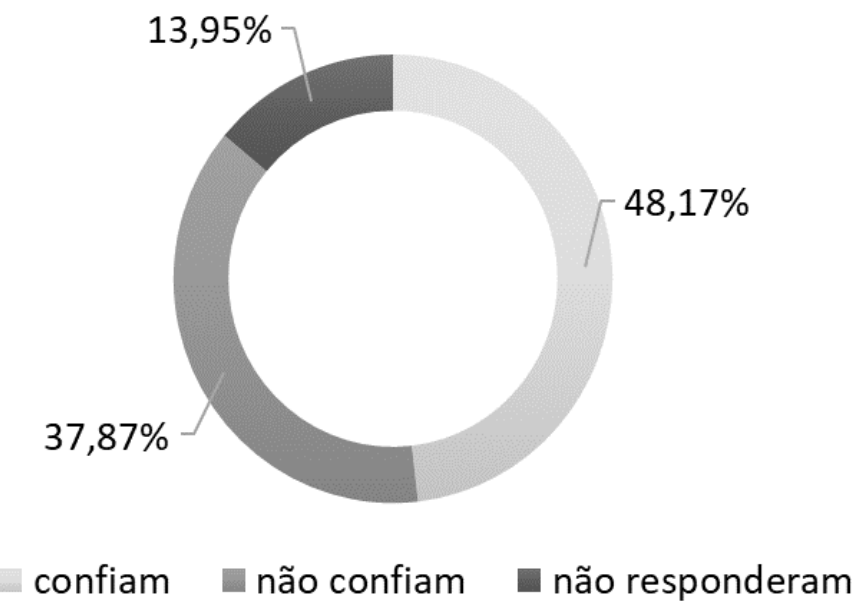

Figura 2- Capacidade de entendimento sobre as informações lidas nos rótulos de produtos alimentícios por consumidores de supermercados da cidade de Niterói-RJ, 2016.

Por isso, a demanda crescente da sociedade por informações confiáveis no que diz respeito aos produtos alimentares exige esforço do governo e setor produtivo para implantação de uma efetiva rotulagem nutricional de alimentos (MARINS et al., 2008). 
Com relação as informações mais procuradas no rótulo de um alimento, segundo consumidores de supermercados do município de Niterói-RJ, o prazo de validade do alimento tem grande destaque $(42,53 \%)$ no momento da escolha de compra de um produto, frente as demais informações, tais como tabela nutricional $(20,29 \%)$ e lista de ingredientes $(19,43 \%)$, conforme ilustrado na Figura 4.

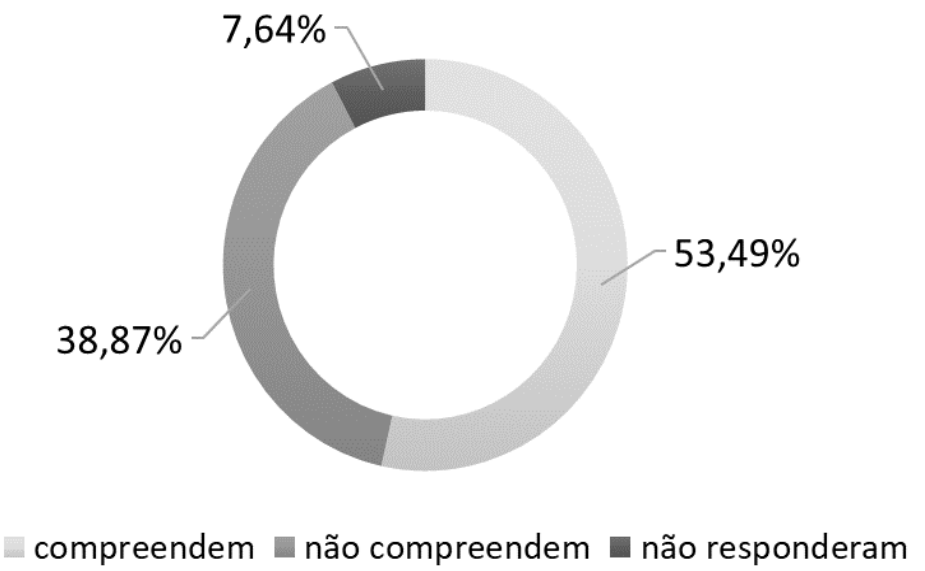

Figura 3- Confiabilidade sobre a veracidade das informações descritas na embalagem de produtos alimentícios, por consumidores de supermercados da cidade de Niterói-RJ, 2016.

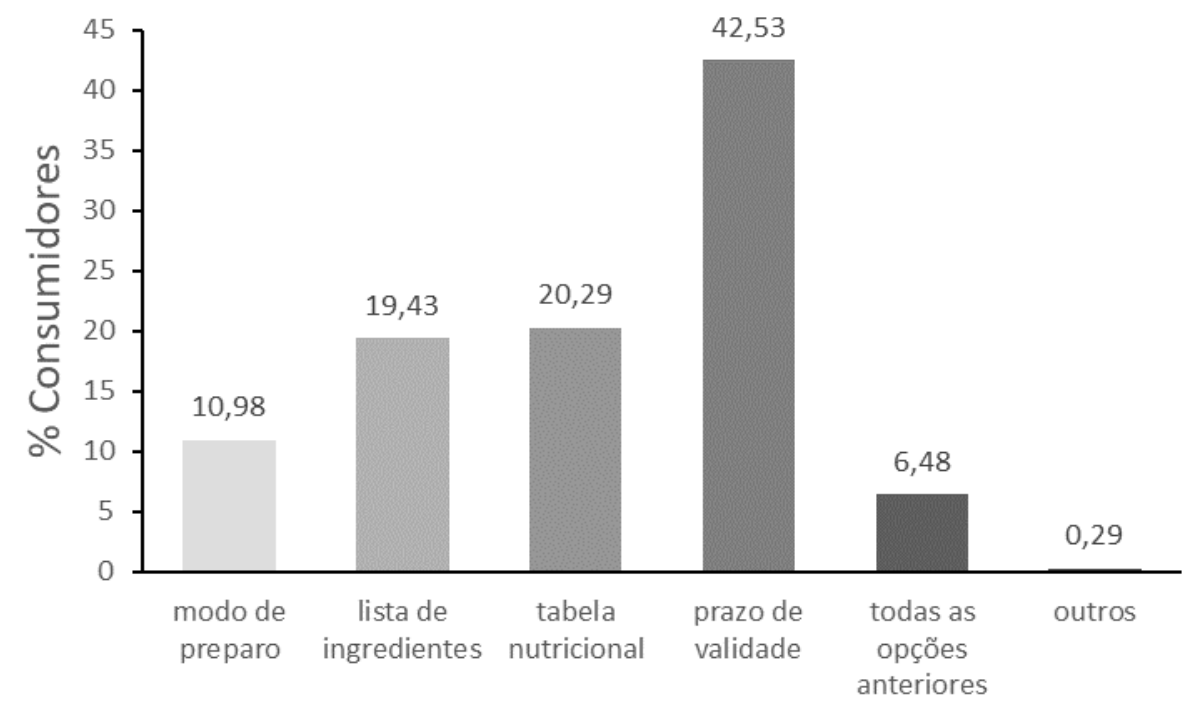

Figura 4- Itens observados nos rótulos de produtos alimentícios, no momento da compra, por consumidores de supermercados da cidade de Niterói-RJ, 2016

É importante destacar que a centralidade do uso e do entendimento da rotulagem nutricional, ressaltada pelos entrevistados, está no prazo de validade e no modo de conservação dos alimentos, sobrepondo-se ao entendimento do valor nutricional em si, que é um dos principais objetivos da rotulagem nutricional obrigatória. Este fato evidencia a dificuldade de compreensão das informações 
nutricionais contidas nas embalagens, demonstrando uma contradição quanto à finalidade da rotulagem de alimentos, tendo em vista que as informações presentes no rótulo deveriam descrever as características do produto com o intuito de informar o consumidor sobre o que ele pretende adquirir, mas que acaba por distanciá-lo devido à ausência de uma informação clara e acessível à população.

Por outro lado, ainda pode ser observada a importância atribuída à presença das informações nos rótulos, principalmente do prazo de validade, relevante para $42,53 \%$ dos consumidores com o hábito da leitura dos rótulos de alimentos, evidenciando, em alguns discursos, a utilização desta informação como fator de grande preocupação em não comprar alimentos "estragados". Em outros estudos (SOUZA et al., 2011; JACOBS et al., 2010), o prazo de validade é também o item que mais se destaca dentre os mais utilizados nos rótulos, auxiliando a verificar a qualidade do produto que se deseja adquirir.

Segundo àqueles participantes que praticam a leitura das informações descritas no rótulo de um produto alimentício, esta prática não tem relação com possíveis problemas de saúde $(55,81 \%)$ para a maioria dos entrevistados (Figura $5 \mathrm{~A})$, enquanto que $28,90 \%$ dos consumidores realizam a leitura do rótulo preocupados com possíveis causas de doenças crônicas não-transmissíveis, dentre estas causas associadas estão hipertensão (28,74\%), diabetes (21,84\%), alergia/restrição alimentar (21,83\%), dentre outras (Figura 5B), como fatores interferentes na escolha de um alimento.

A

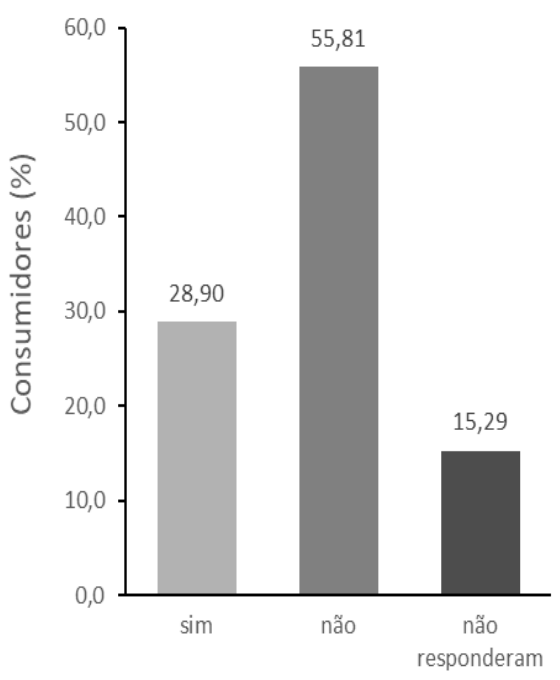

B

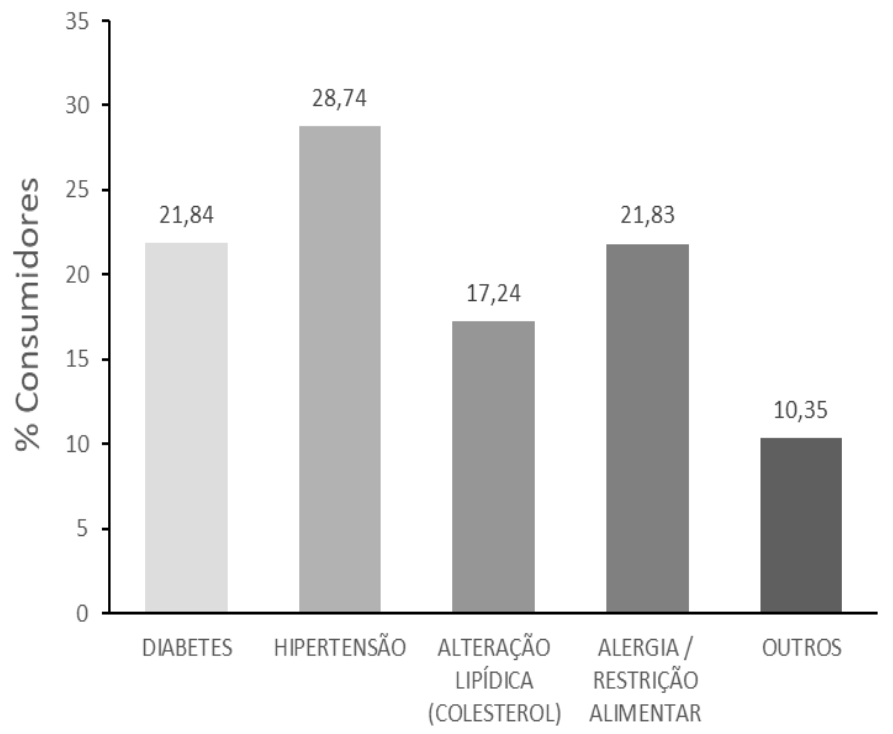

Figura 5 Verificação entre uma possível correlação entre o hábito de leitura do rótulo e problemas de saúde $(5 \mathrm{~A})$. Causas associadas à prática da leitura dos rótulos de alimentos (5B).

Avaliou-se, também, a importância dos nutrientes descritos na tabela nutricional de alimentos embalados, segundo os consumidores das redes de supermercados do município de Niterói-RJ. De acordo com os entrevistados, o 
sódio é o nutriente mais relevante $(24,10 \%)$, seguido do valor calórico $(19,42 \%)$ e carboidratos $(15,85 \%)$, como mostrado na Figura 6.

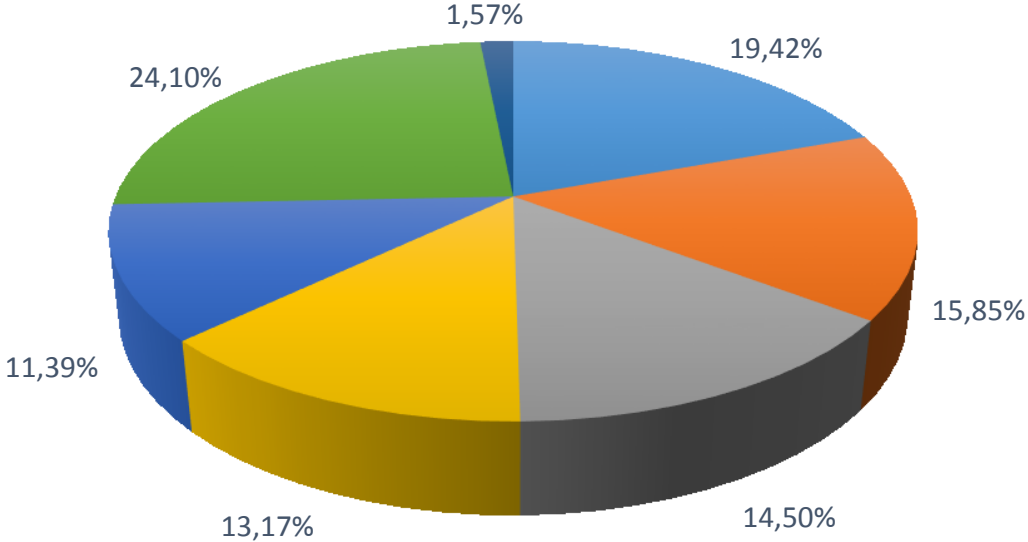

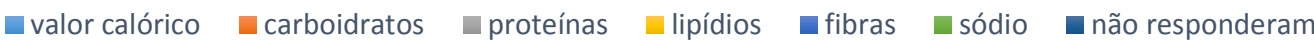

Figura 6- Nutrientes da tabela de informação nutricional considerados mais importantes segundo os consumidores de supermercados do município de Niterói-RJ

(2016)

Dentre os elementos nutricionais mais buscados, esse estudo, também revelou a preocupação dos consumidores com a aquisição de alimentos ricos em calorias, carboidratos e sódio. Assim, a declaração nutricional subsidiou o controle dietoterápico para determinados tipos de doenças crônicas não transmissíveis, como diabetes e hipertensão, conforme ilustrado na Figura 6, de acordo com informações cedidas pelos entrevistados. Este dado pode estar ainda relacionado à preocupação crescente com a aparência corporal e à insatisfação generalizada com o corpo, veiculada principalmente pelas grandes mídias.

Um fator que a indústria utiliza para incentivar a aquisição de alimentos industrializados é o marketing, que pode apresentar-se em embalagens, rádios, televisão e mercados. Com o objetivo de estimular a compra e ingestão de alimentos industrializados, as técnicas de marketing utilizadas pelas indústrias podem ir contra a legislação, com a utilização de parâmetros nutricionais incorretos. Este comportamento torna-se mais evidente ao considerar que grupos de pessoas estão alterando os hábitos alimentares de maneira equivocada, em consequência de índices de saúde preocupantes que estão intimamente relacionados à má alimentação (VICENTINI, 2015).

Porém, para a maior parte do público participante desse estudo (63,46\%), o marketing não tem influência sobre a decisão de compra de um produto. No entanto, $36,54 \%$ dos entrevistados consideram o marketing como um dos principais fatores determinantes na seleção de um alimento embalado. 0 entendimento prévio sobre o papel dos rótulos de alimentos poderia permitir aos consumidores prestar mais atenção a informações relevantes sobre um rótulo de alimento, e ignorar características de marketing que não refletem qualidades nutricionais significativas (MILEER; CASSADY, 2015). 
Nesse estudo, os consumidores reconheceram que as informações descritas nos rótulos de alimentos são necessárias e devem ser levadas em consideração, tomando-as como um instrumento fundamental de educação nutricional. Contudo, os resultados obtidos sugerem a necessidade de implantação de programas comunitários de saúde e nutrição, que devem estimular a leitura e utilização das informações descritas nos rótulos de alimentos, promovendo práticas que auxiliam a população no entendimento e intepretação das informações fornecidas.

\section{CONCLUSÕES}

A educação em saúde consiste no processo que capacita os indivíduos a agir conscientemente diante da realidade cotidiana, com aproveitamento de experiências anteriores, tendo sempre em vista a integração do conhecimento e o progresso no âmbito social (SALCl et al., 2013). Assim, a educação nutricional é um processo de grande importância na promoção de práticas saudáveis e na modificação de hábitos alimentares. Neste contexto, os rótulos de alimentos vêm sendo desenvolvidos como veículo de informação ao consumidor.

A partir dos dados obtidos neste estudo conclui-se que a maioria dos consumidores possuía o hábito de leitura sobre os rótulos dos alimentos consumidos e, além disso, dos entrevistados que consultavam os rótulos, pouco mais da metade compreendia as informações lidas. Os dados também mostraram uma preocupação dos consumidores quanto as informações nutricionais, sendo consideradas relevantes, pelos participantes, informações como valor calórico, conteúdo de carboidratos e sódio. E, ainda, de acordo com este estudo, o marketing não tem grande influência na decisão de compra de um produto alimentício.

No entanto, as informações descritas nos rótulos de alimentos não são de tão simples entendimento para os consumidores, o que demonstra a necessidade de ações educativas em relação à rotulagem nutricional, bem como a elaboração de rótulos com informações claras e objetivas, que facilitem a compreensão do consumidor. 


\title{
Evaluation of comprehension on labeling of packed food products according to consumers in Niterói, Rio de Janeiro, Brazil
}

\begin{abstract}
The present study aimed to evaluate consumers' understanding of the information contained on labels of the food products marketed in supermarkets in the city of Niterói, Rio de Janeiro, Brazil. During 90 days of 2016, 301 consumers were interviewed through a structured form about their understanding of nutritional labeling. The results showed that $63.79 \%$ of the interviewees read the food labels, of which only $53.49 \%$ understood the information read. Participants, who had no custom to read the food label, stated that lack of habit and illegible handwriting are two important points for non-practice of nutritional reading. For those interested in the label, the shelf life and nutritional table are the most read information on the product packaging. Consumers showed greater concern about information such as caloric value, carbohydrate and sodium content; these data are related to the search for nutritional information due to health problems, such as diabetes and hypertension, as reported by participants. Moreover, for $63.46 \%$ of consumers, in this study, marketing has no bearing on the decision to purchase a packaged product. It is concluded that the information contained in the food labels is relevant for the general public, but there is a need for educational projects in the nutritional scope, in order to facilitate the understanding and better use of the information described in the food packaging.
\end{abstract}

KEYWORDS: Nutrition labwling; consumer behavior; nutrition education. 


\section{REFERÊNCIAS}

BRASIL. Ministério da Saúde (BR). Agência Nacional de Vigilância Sanitária. Resolução RDC ANVISA/MS n 259, de 20 de setembro de 2002. Regulamento técnico para rotulagem de alimentos embalados. Disponível em: http://http://portal.anvisa.gov.br/documents/10181/2718376/RDC_259_2002_C OMP.pdf/9c816a4d-2dc7-48bf-80e4-e8891f640cf2 . Acesso em 20.10.2017.

BRASIL. Ministério da Saúde. Agência Nacional de Vigilância Sanitária Rotulagem nutricional obrigatória: manual de orientação às indústrias de Alimentos. 2a versão. Brasília: Universidade de Brasília, 2005b.

CASSEMIRO, I.A.; COLAUTO, N.B.; LINDE. G.A. Rotulagem nutricional: quem lê e por quê? Arquivos de Ciências da Saúde da UNIPAR, v. 10, n. 1, p. 9-16, 2006.

COLBY, S.E.; JOHNSON, L.; SCHEETT, A.; HOVERSON, B. Nutrition marketing on food labels. Journal of Nutrition Education and Behavior, v. 42, n. 2, p. 92-98, 2010.

JACOBS, S.A.; BEER, H.; LARNEY, M. Adult consumers' understanding and use of information on food labels: a study among consumers living in the Potchefstroom and Klerksdorp regions, South Africa. Public Health Nutrition, v. 14, n. 3, p. 510$522,2010$.

LOBANCO, C.M.; VEDOVATO, G.M.; CANO, C.B.; BASTOS, D.H.M. Fidedignidade de rótulos de alimentos comercializados no município de São Paulo, SP. Revista de Saúde Pública, v. 43, n. 3, p. 499-505, 2009.

MACHADO, S.S.; SANTOS, F.O.; ALBINATI, F.L.; SANTOS, L.P.R. Comportamento dos consumidores com relação à leitura de rótulo de produtos alimentícios. Alimentos e Nutrição Araraquara, vol. 17, n. 1, p. 97-103, 2006.

MARINS, B.R.; JACOB, S.C.; PERES, F. Avaliação qualitativa do hábito de leitura e entendimento: recepção das informações de produtos alimentícios. Ciência e Tecnologia de Alimentos [online], v. 28, n. 3, p. 579-585, 2008.

MILLER, L.M.S.; CASSADY, D.L. The effects of nutrition knowledge on food label use. A review of the literature. Appetite, v. 92, n. 1, p. 207-216, 2015.

MONDINI, L.; MONTEIRO, C.A. Mudanças no padrão de alimentação da população urbana brasileira (1962-1988). Revista de Saúde Pública, v. 28, n. 6, p. 433-439, 1994. 
PONTES, T.E.; COSTA, T.F.; MARUM, A.B.R.F.; BRASIL, A.L.D.; TADDEI, J.A.A.C. Orientação nutricional de crianças e adolescentes e os novos padrões de consumo: propagandas, embalagens e rótulos. Revista Paulista de Pediatria [online], v. 27, n. 1, p. 99-105, 2009.

SALCI, M.A.; MACENO, P.; ROZZA, S.G.; SILVA, D.M.G.V.; BOEHS, A.E.; HEIDEMANN, I.T.S.B. Educação em saúde e suas perspectivas teóricas: algumas reflexões. Texto \& Contexto - Enfermagem, v. 22, n. 1, p. 224-230, 2013.

SANTOS, L.A.S. Educação alimentar e nutricional no contexto da promoção de práticas alimentares saudáveis. Revista de Nutrição, v.18, n. 5, p. 681-692, 2005.

SILVA, M.Z.T. Influência da Rotulagem na Rotulagem Nutricional sobre o Consumidor. 67 f. Dissertação (Mestrado em Nutrição) - Universidade Federal de Pernambuco, Recife, 2003.

SIQUEIRA, R.S.S.; HAESE, T.D.P.; CARDOSO, C.; MACIEL, JR.; PIMASSONI, L.H.S.; MORO, A.S.; SILVA-SENA, G.G. Avaliação do entendimento e da atitude do consumidor diante das informações veiculadas na rotulagem de produtos alimentícios na Grande Vitória, Espírito Santo. Nutrire, v. 39, n. 2, p. 214-221, 2014.

SOUZA, S.M.F.C.; LIMA, K.C.; MIRANDA, H.F.; CAVALCANTI, F.I.D. Utilização da informação nutricional de rótulos por consumidores de Natal, Brasil. Revista Panamericana de Salud Pública, v. 29, n. 5, p. 337-343, 2011.

SOUZA, S.M.F.C.; LIMA, K.C.; ALVES, M.S.C.F. A rotulagem nutricional para escolhas alimentares mais saudáveis: estudo de intervenção, Natal - RN. Vigilância Sanitária em Debate, v. 2, n. 1, p. 64-68, 2014.

VICENTINI, M.S. Alimentos industrializados: abordagem da indústria, consumidores e governo. Segurança Alimentar e Nutricional, v. 22, n. 1, p. 671$682,2015$.

YOSHIZAWA, N.; POSPISSIL, R.T.; VALENTIM, A.G.; SEIXAS, D.; ALVES, F.S.; CASSOU, F.; YOSHIDA, I.; SEGA, R.A.; CÂNDIDO, L.M.B. Rotulagem de alimentos como veículo de informação ao consumidor: adequações e irregularidades. Boletim Centro de Pesquisa de Processamento de Alimentos, v. 21, n.1, p. 169180, 2003. 
Recebido: 05 dez. 2017

Aprovado: 30 nov. 2018

DOI: $10.3895 /$ rebrapa.v9n4.7477

Como citar:

LIBERTI, P. et al. Avaliação da compreensão de rótulos de alimentos embalados por consumidores do município de Niterói, Rio de Janeiro, Brasil. Brazilian Journal of Food Research, Campo Mourão, v. 9, n. 4 p. 61-73, out./dez. 2018. Disponível em: https://periodicos.utfpr.edu.br/rebrapa

\section{Correspondência:}

Nathalia Soares

Departamento de Nutrição, Bromatologia e Tecnologia de Alimentos, Centro Universitário Anhanguera de Niterói, Rio de Janeiro, Brasil.

Direito autoral: Este artigo está licenciado sob os termos da Licença Creative Commons-Atribuição 4.0 Internacional. 